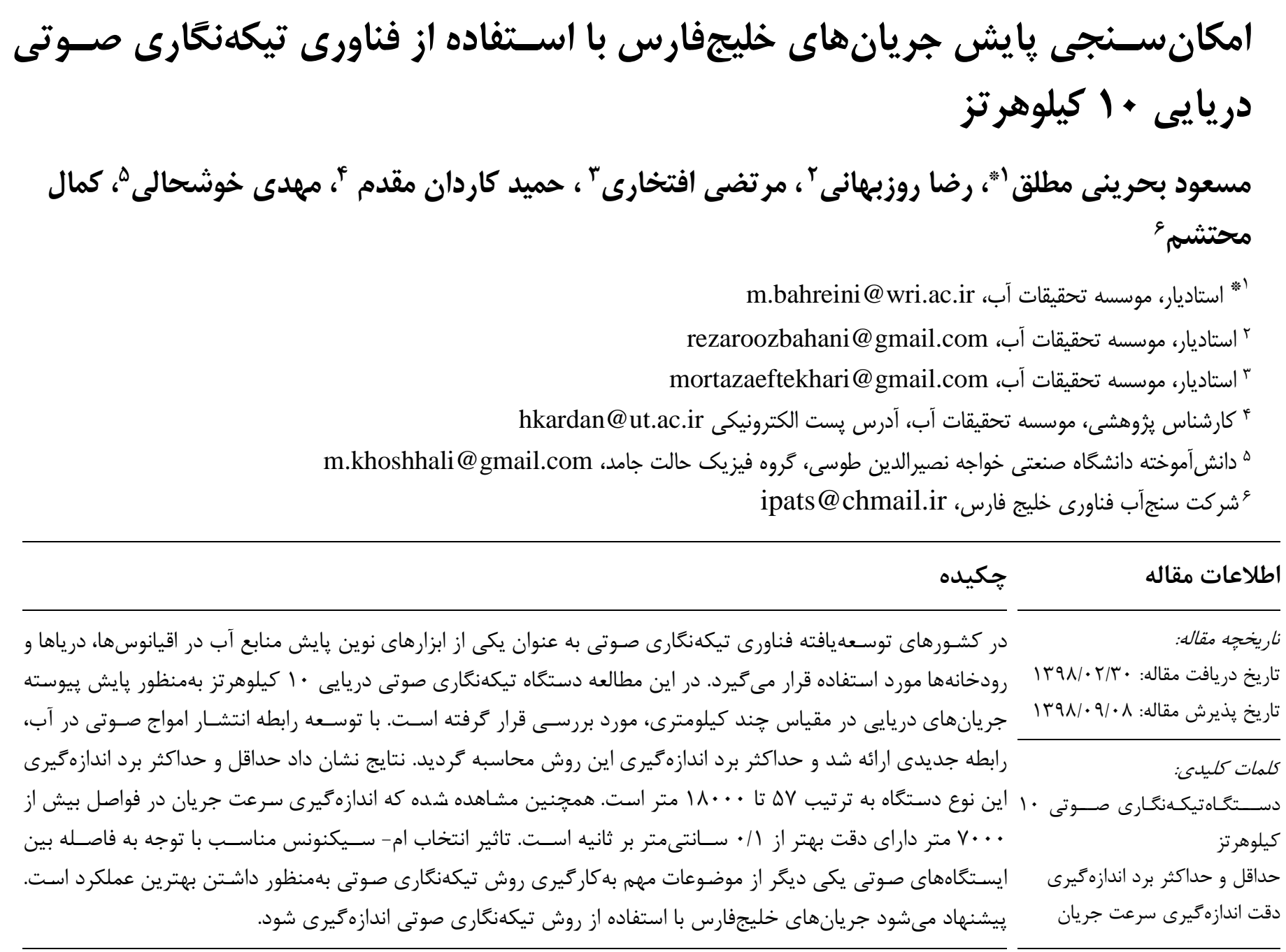

\title{
Feasibility study of 10-kHz Coastal Acoustic Tomography System for current monitoring in the Persian Gulf
}

\section{Masoud Bahreinimotlagh ${ }^{*}$, Reza Roozbahani ${ }^{2}$, Mortaza Eftekhari ${ }^{3}$, Hamid Kardanmoghadam $^{4}$, Mahdi Khoshhali ${ }^{5}$, Kamal Mohtasham ${ }^{6}$}

$I^{*}$ Assistant Professor, Water Research Institute; m.bahreini@wri.ac.ir

${ }^{2}$ Assistant Professor, Water Research Institute; rezaroozbahani@gmail.com

${ }^{3}$ Assistant Professor, Water Research Institute; mortazaeftekhari@gmail.com

${ }^{4}$ Scientific researcher, Water Research Institute; hkardan@ut.ac.ir

${ }^{5}$ Graduated of Solid Physics, K. N. Toosi University of Technology; m.khoshhali@gmail.com

${ }^{6}$ Sanjab Fanavari Khalije Fars Ltd; ipats@chmail.ir

\section{ARTICLE INFO}

Article History:

Received: 20 May. 2019

Accepted: 29 Nov. 2019

\section{Keywords:}

10-kHz Coastal Acoustic

Tomography Technique

Minimum and Maximum

Operational Ranges

Velocity Resolution

\begin{abstract}
Acoustic Tomography (AT) technique is widely used in developed countries for water resources monitoring of the oceans, seas, and rivers. The 10-kHz Coastal Acoustic Tomography System (CATS) is previously applied to monitor coastal seas in the scale of kilometers. In this study, we evaluated the capability of the $10-\mathrm{kHz}$ CAT system. The equation of sound propagation in water was developed to introduce a new equation to estimate the maximum operational range of 10$\mathrm{kHz}$ CAT system. The results showed that with the assumption of clear seawater, the minimum and maximum operational ranges are 57 and $18000 \mathrm{~m}$, respectively. Moreover, the velocity resolution is better than $0.1 \mathrm{~cm} / \mathrm{s}$ in the range of greater than $7000 \mathrm{~m}$. Choosing a suitable M Sequence due to the distances between acoustic stations is another point of operation to have the best performance. It is suggested that scientists apply this technique to monitor the Persian Gulf currents.
\end{abstract}


اى إوىامَّ (با بسـامدهاى هץ كيلوهرتز تا ب مكاهرتز)، قابليت انتشـار

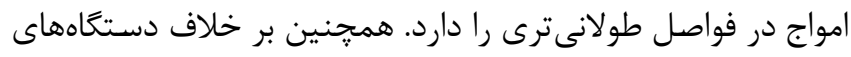

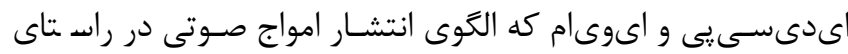

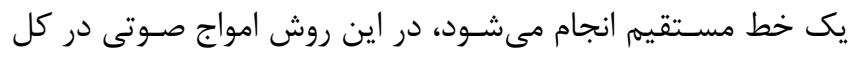

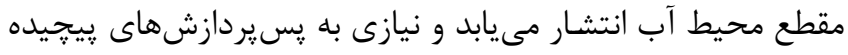

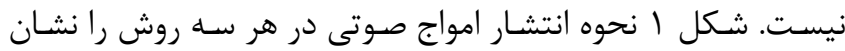

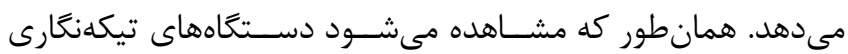

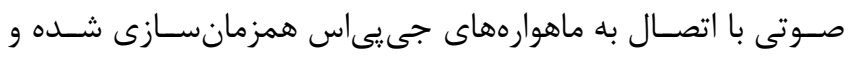

امواج صوتى را در كل مقطع محيط آب انتشار مىدهند [10].

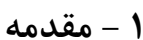

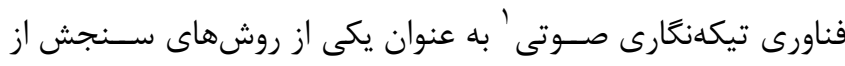

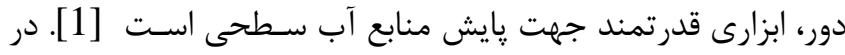

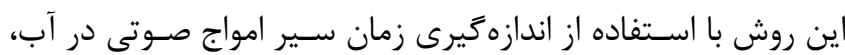

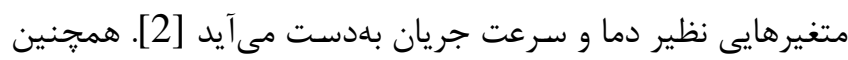
از روش تيكهنغارى صوتى جهت صحتسنجى مدل هاى انتشار امواج

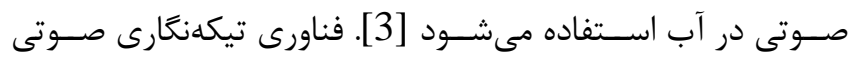

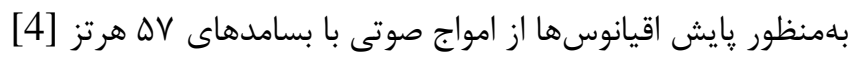

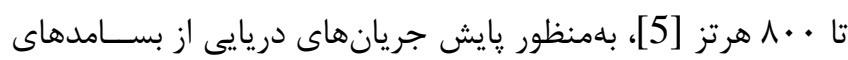

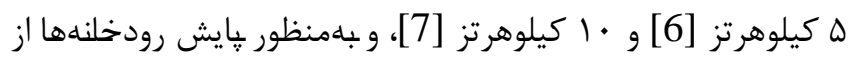

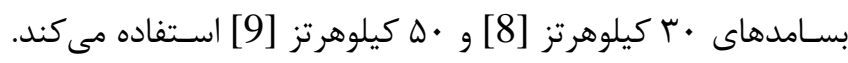

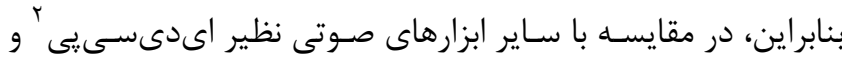

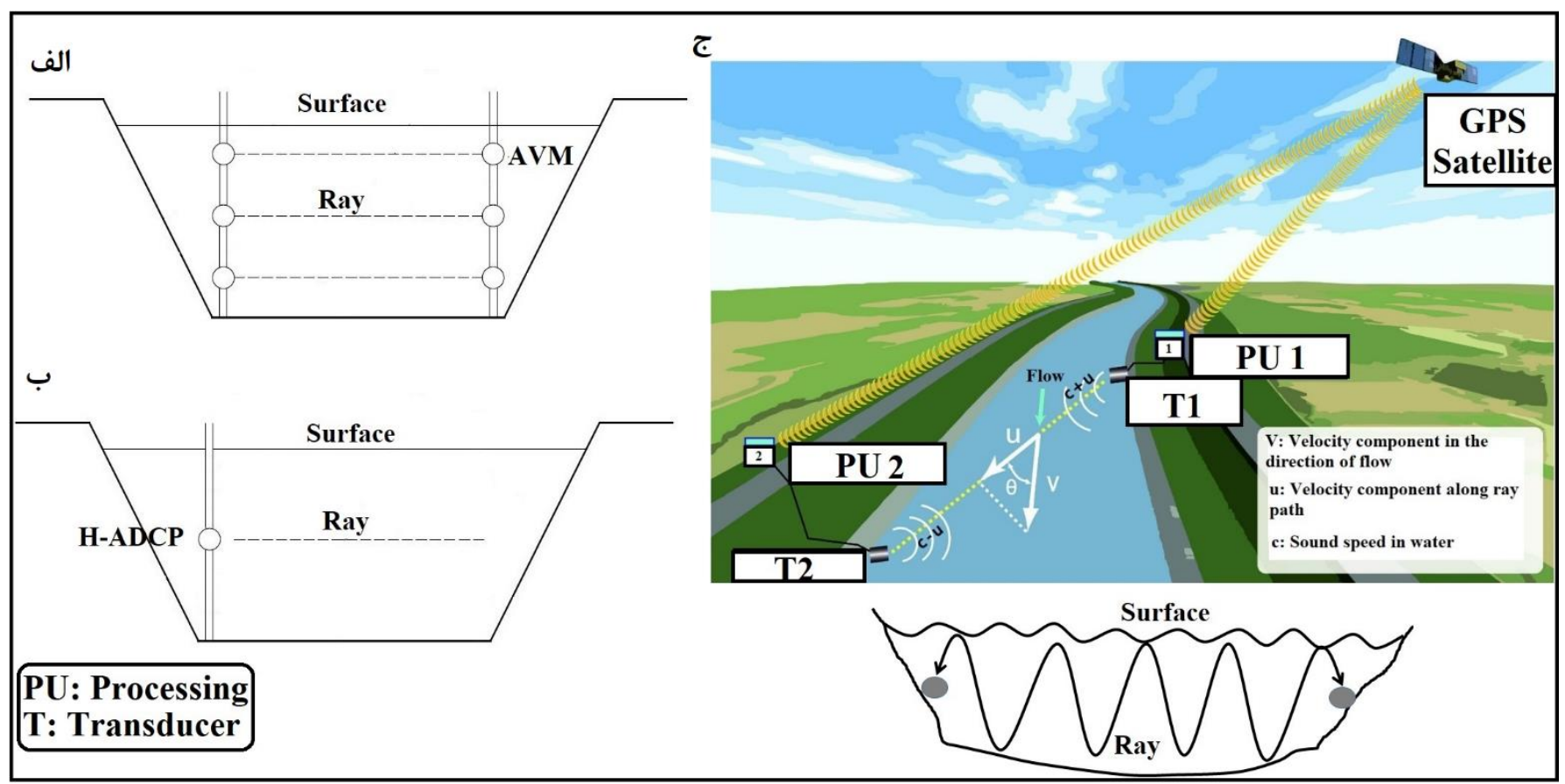

شكل ا - الكَوى انتشار امواج صوتى با ابزارهاى الف) ایىوىام، ب) اىدىسى يى افقى، و ج) تيكهنكًارى صوتى [10].

زإين را به مدت ه سـاعت اندازمخيرى كردند. دو ايستَكاه صـوتى به

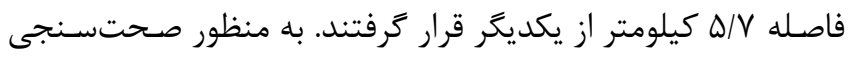

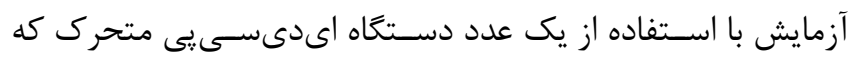
توسط كشتى در راستاى ارسال امواج صوتى دستخاههاى تيكهنغارى

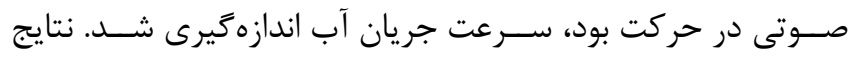

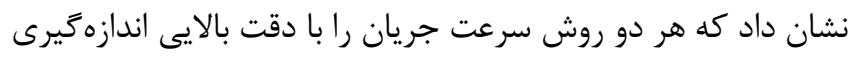

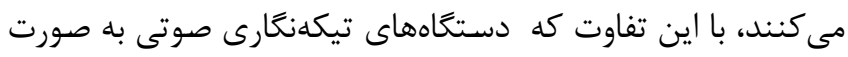

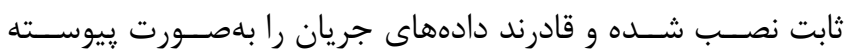

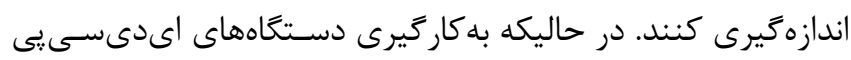

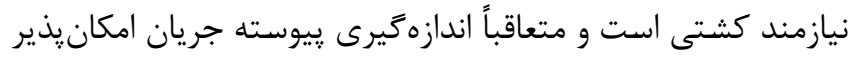

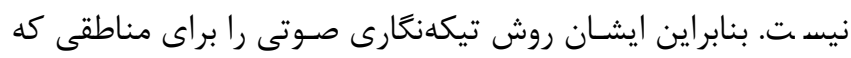

روش تيكهنكارى صـوتى توســ مونك و همكاران 9 و بهمنظور

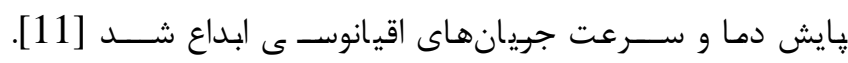

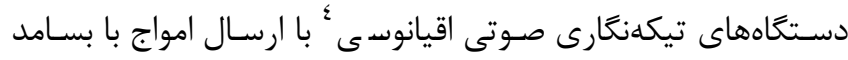

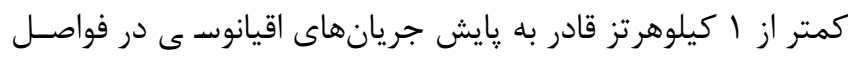

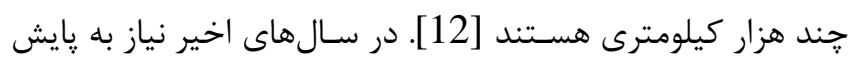

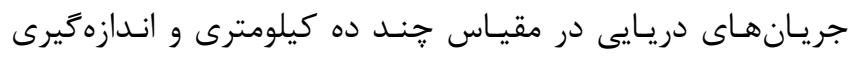

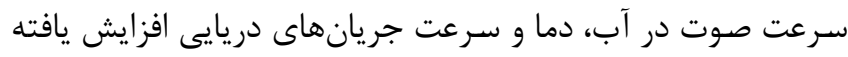

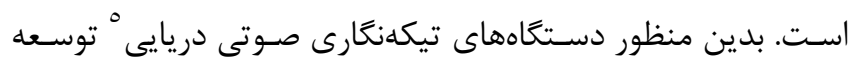

يافتهاند [13].

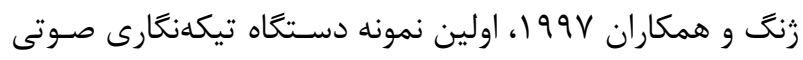

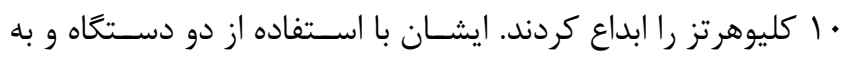

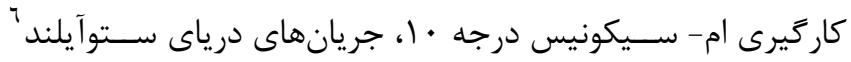


داد كه دستَاه تيكهنغارى صوتى بهمنظور پايش جريان در اسكلهها

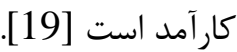

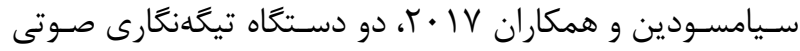

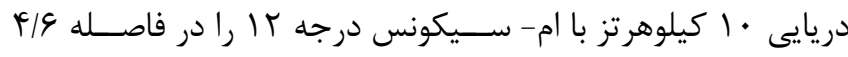

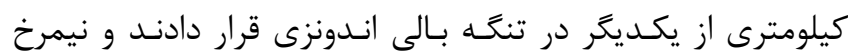
عمودى جريانهاى جزر و مدى را لندازمخيرى كرسند. نتايج نشــان

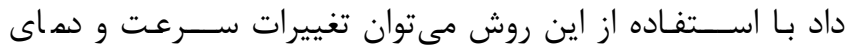

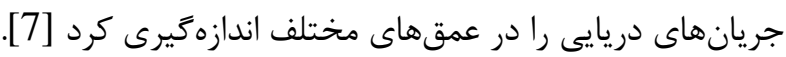

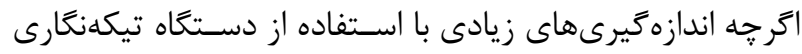
صوتى دريايى • ا كيلوهرتز در مناطق مختلف انجام شـده است، اما

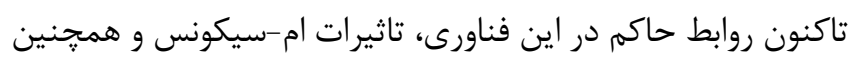

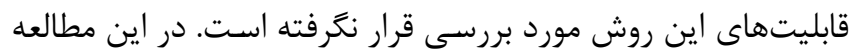

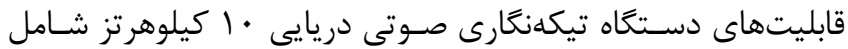

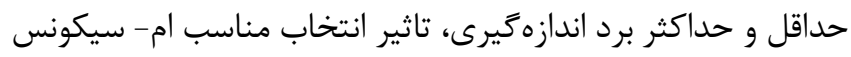

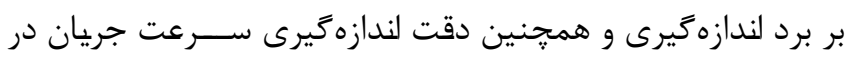
فواصل مختلف مورد بررسى قرار مى گيرد.

\section{Y - روابط اساسى حاكم در روش تيكهنكارى صوتى}

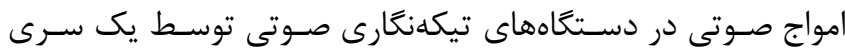

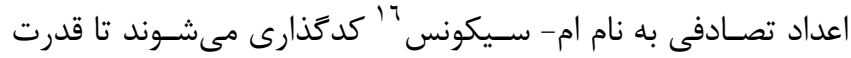

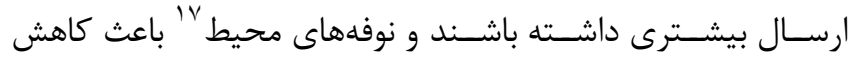
سيكنالهاى صوتى نشوند [20]. دستَاههاى تيكدنغارى صوتى از

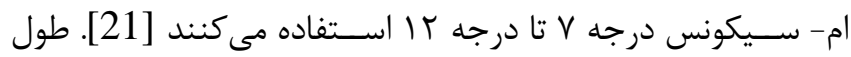

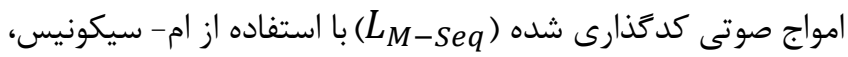

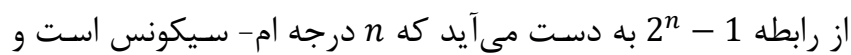

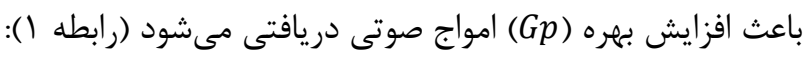

$$
G p=10 \log L_{M-S e q}
$$

دسـتخاههاى تيكهنغارى صـوتى، امواج صـوتى كدكذارى شـده با

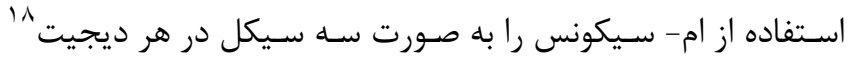

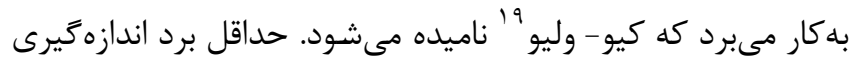

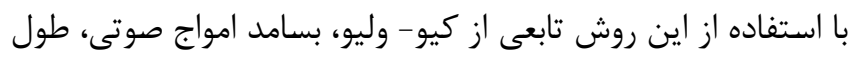

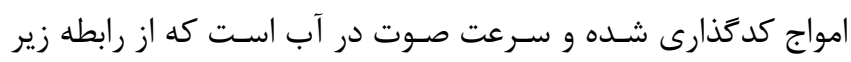

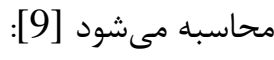

$$
\begin{aligned}
R_{\text {min }}=\frac{Q-\text { value }}{f} & \times L_{M-S e q} \\
& \times c
\end{aligned}
$$

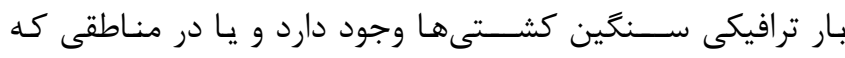
فعاليتهاى ماهيخيرى انجام مىشود توصيه كردند [114].

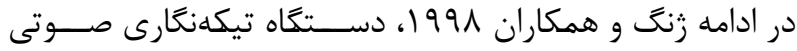

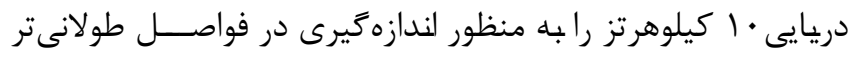

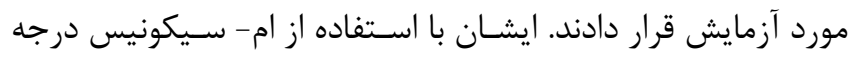

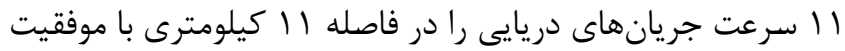

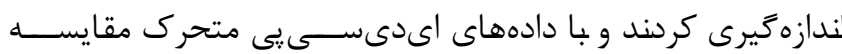

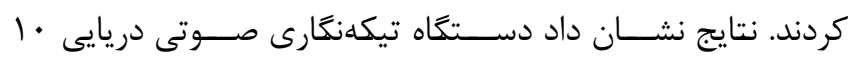

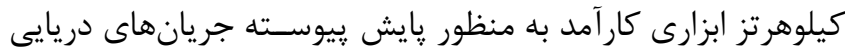
در فواصل جند كيلومترى است [15].

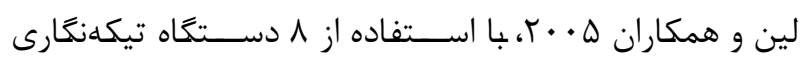

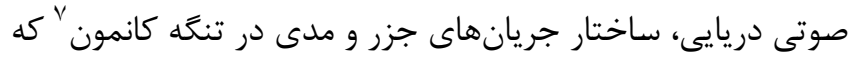

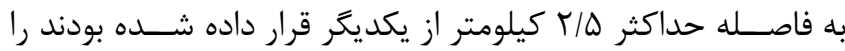

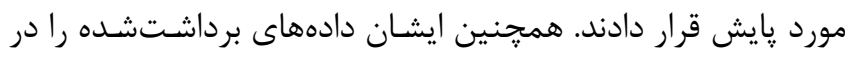

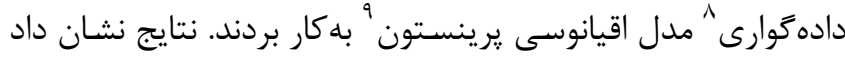

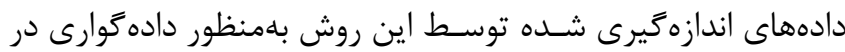
مدلهاى رايانهاى قابل استفاده است. ايشان دستخاه فوق را به دليل نادي

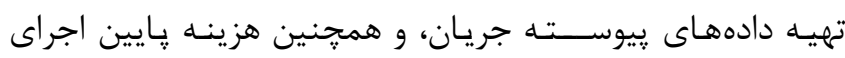
عمليات دادهبردارى توصيه كردند [16].

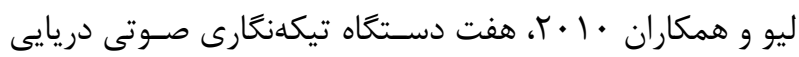

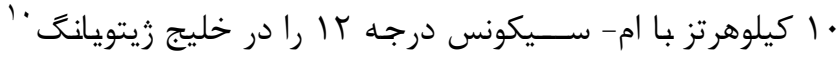

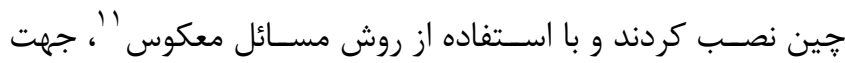

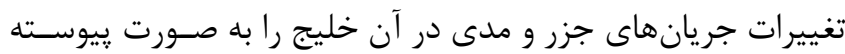

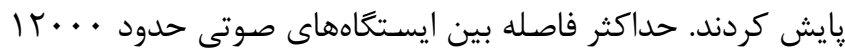

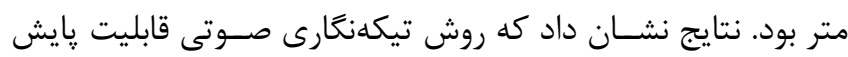
دو-بعدى جريان هاى دريايى را دارد. همجنين اين روش براى بإيش

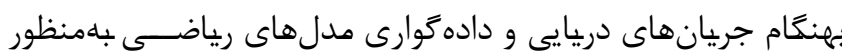

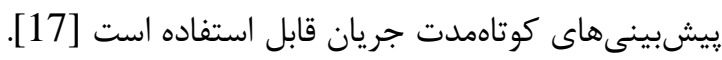

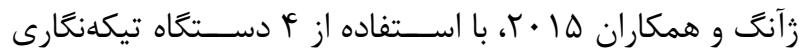

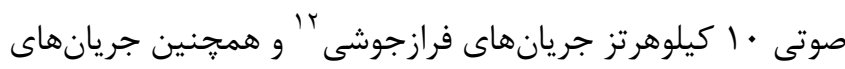

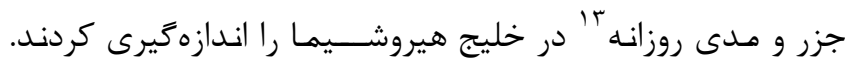

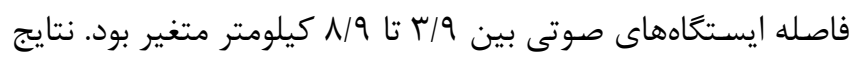
نشان داد روش تيكهنغارى صوتى ابزارى بسيار قدرتمند جهت بإيش

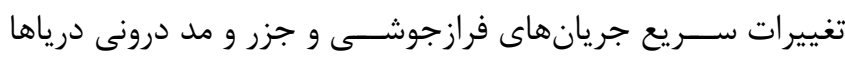

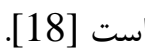

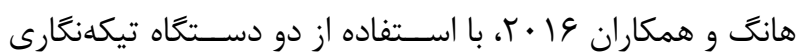

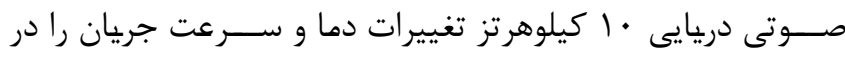

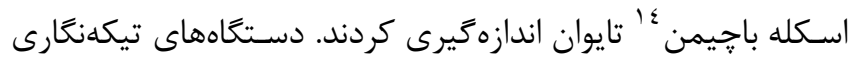
صوتى از ام- سيكونس درجه · ل استفاده كردند و فاصله بين آنها

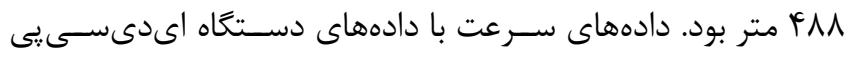

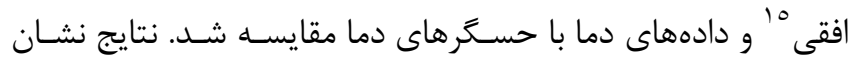




$$
U r=\frac{c^{2}}{2 L} \times \frac{1}{2 f} \times \frac{1}{\sqrt{n}}
$$

كه c سـرعت صوت در آب (متر بر ثانيه)، L فاصله بين دو دستخاه تيكهنغارى صـوتى (متر)، f بسـامد امواج صـوتى (هرتز) و $n$ تعداد دادههاى ميانخين گيرى متحرى است.

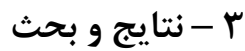

ا-Y حداقل برد اندازهيرى دستخاه تيكهنغارى +ا كيلوهرتز بر اســاس رابطه (؟) حداقل برد اندازهيرى، تابعى از بســامد امواج

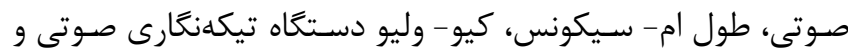

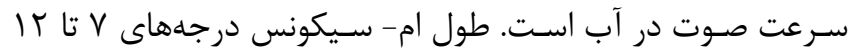
در جدول ا ارائه شده است. همانطور كه مشاهده مىشود كمترين

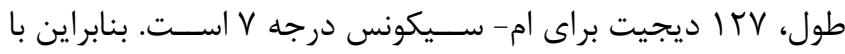

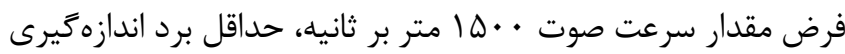

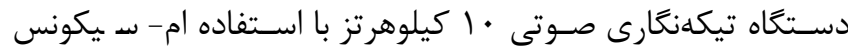

$$
\text { درجه V برابر DV متر است. }
$$

جدول ا - ام - سيكونسهاى قابل استفاده در دستخاهتيكهنگًارى صوتى

\begin{tabular}{cc}
\multicolumn{2}{c}{ M-Sequence Degree } \\
(n)
\end{tabular}$\quad \begin{gathered}\text { Maximum Period (L) } \\
\text { digit }\end{gathered}$

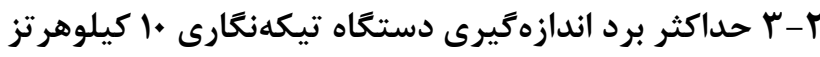
با توجه به رابطه (9)، حداكثر برد اندازهگيرى در شرايط ايدهآل و

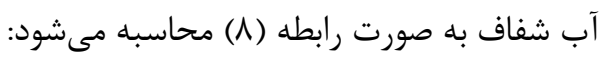

$$
\begin{aligned}
20 \log R_{\max }+ & \alpha R_{\max } \\
& =S L-L_{0}+G p-N a \\
& -S N R_{\text {Threshold }}
\end{aligned}
$$

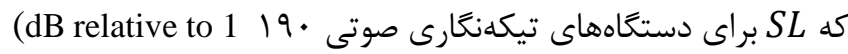

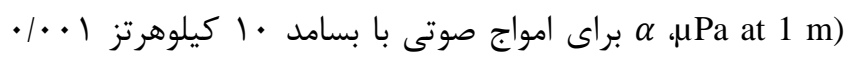
دسىبل بر متر است، $L_{0}$ برابر • دسىبل، و و Gp براى امسيكونسهاى مختلف در جدول r ارائه شده است. بنابراين براى

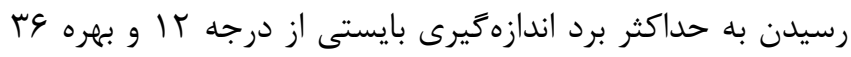

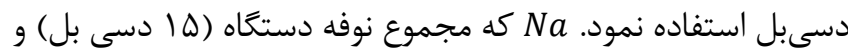
نوفه محيط در شرايط ترافيك سنَّين عبور كشتىها (ه دسى بل)

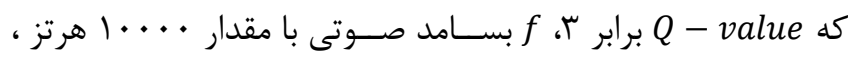

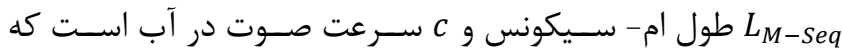

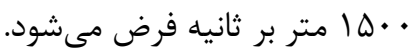

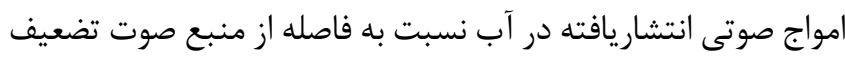

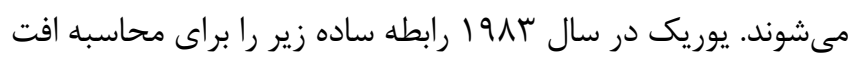
انتشار ‘ 'امواج صوتى نسبت به فاصله از منبع صوتى ارائه كرد [22]:

$$
P L=20 \log R+\alpha R+L_{0}
$$

كه به ترتيب جملات اول و دوم در سمت راست رابطه مربوط به

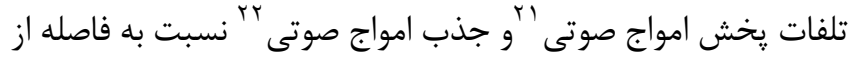
منبع صوتى (R) است. همجنين

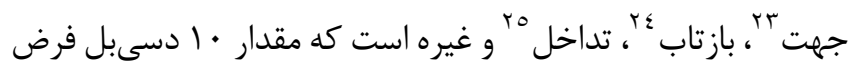
مىشود. ضريب جذب امواج صوتى (م) در آب شفاف (با فرض عدم وجود ذرات معلق) از رابطه زير به دست مى آيد [23]

$$
\begin{aligned}
& \alpha_{w}=\alpha(\mathrm{dB} / \mathrm{m}) \\
& =\left(3.3 \times 10^{-3}+\frac{0.11 f^{2}}{1+f^{2}}+\frac{44 f^{2}}{4100+f^{2}}+3 \times 10^{-4} f^{2}\right) /_{1000}
\end{aligned}
$$

كه f بسامد امواج صوتى به هرتز است.

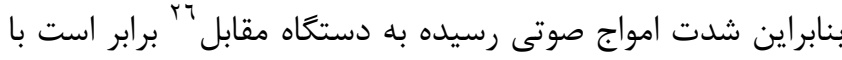

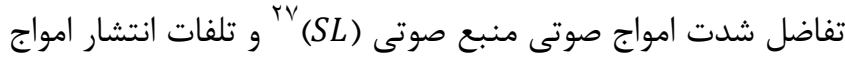

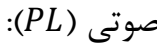

$$
R L=S L-P L
$$

رابطه ه براى دستعاههاى تيكهنگارى صوتى بهصورت زير بازنويسى

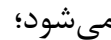

$$
S N R=S L-20 \log R-\alpha R-L_{0}+G p-N a
$$

كه Na مجموع نوفه محيط و دستخاه تيكهنگارى صوتى، Gp بهره دستخاه، SL شدت تراز دستخاه تيكدنگارى صوتى بر حسب relative to 1 rPa at 1 m به نوفه محيط يا همان RL است. دقت اندازهيرى سرعت جريان با استفاده از روش تيكهنغارى صوتى تابعى از سرعت صوت در آب، فاصله بين دستخاههاى

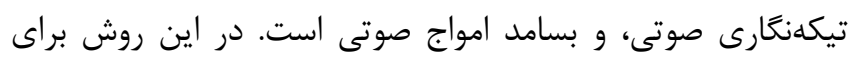
افزايش دقت اندازهخيرى ميانگين متحرى جند وند داده انجام مى

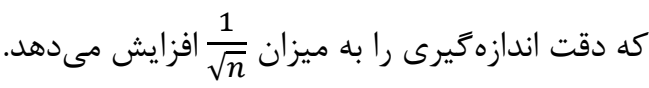


افزايش قابل توجهاى مى يابد، به طورى كه در فاصله . . . 1 مترى

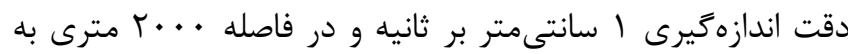
| • • سانتى متر برثانيه مىرسد.

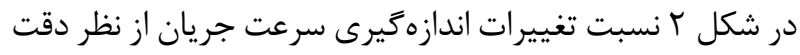

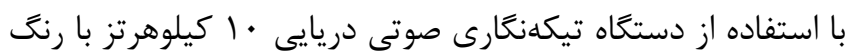
آبى نشان داده شده است. نتايج ارائه شده نشان مىدهد كه رابطهى بين دقت اندازهخيرى نسبت به فاصله دستخاهها بصورت نمايى تغيير

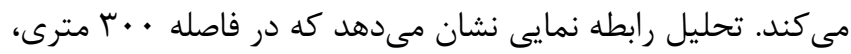
دقت اندازهگيرى اين روش ₹ سانتىمتر بر ثانيه است، درحاليكه اگر

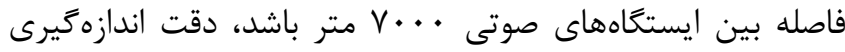
سرعت جريان بهتر از / / • سانتىمتر بر ثانيه خواهد بود. همجنين

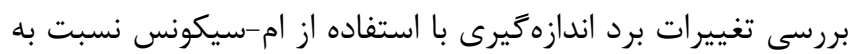

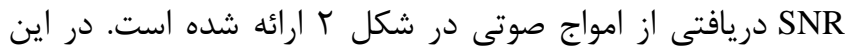

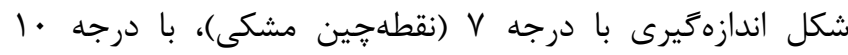

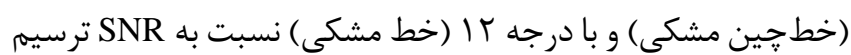

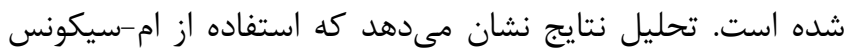

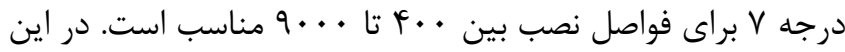

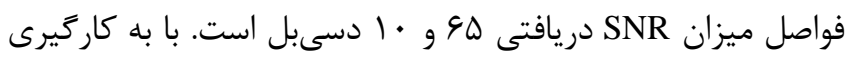
ام- سيكونس درجه • ا، بهترين فاصله نصب ايستخاههاى صوتى به

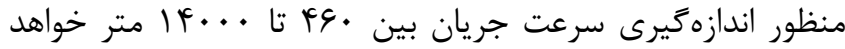
بود. ميزان SNR دريافتى در اين حالت بين وه تا • ا دسى دسيل است.

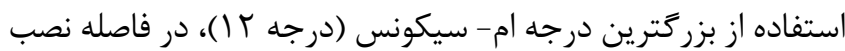

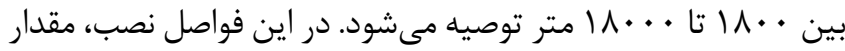
حداكثر SNR دريافتى به ترتيب ^^| و • ا دسىبل بدست آمده است.

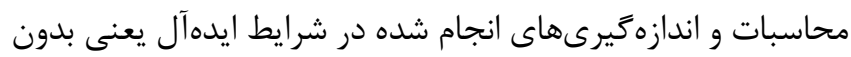
وجود مواد معلق انجام شده است. وجود هرگَونه مواد معلق و ناخالصى سبب كاهش برد اندازهخيرى خواهد شد.

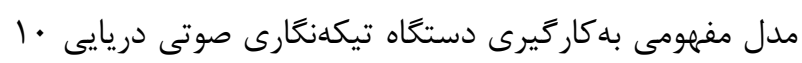
كيلوهرتز در شكل ب نشان داده شده است. همانطور كه مشاهده

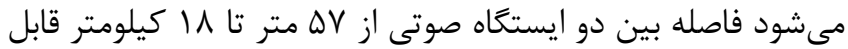

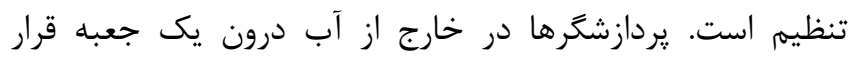

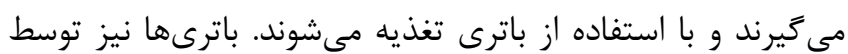
صفحات خورشيدى شارز مى گردند. تراگذارها (ترانسديوسر) توسط يك كابل به يردازش گرها متصل شده و درون آب قرار مى ميرند. همجنين تراگذارها توسط يك وزنه سنگين به كف دريا متصل مىشوند و در نهايت امواج صوتى به سمت يكديگر ارسال مىشوند.

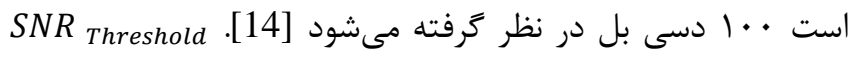
كمترين نسبت سيخَالهاى دريافتشده به نوفه محيط است كه دستخاههاى تيكدنغارى صوتى مىتوانند سيخنال هاى رسيده شده از

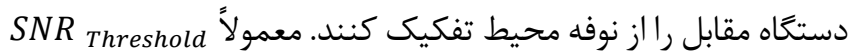

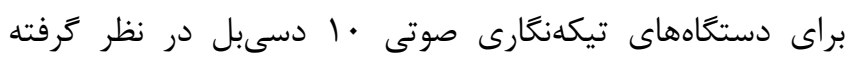

مى شوند [6].

جدول ץ - ام - سيكونسهاى قابل استفاده در دستكاهتيكهنغارى صوتى و افزايش بهره سيخنال هاى صوتى

\begin{tabular}{cc}
\hline M-Sequence Degree (n) & Gain (Gp) dB \\
\hline 7 & 21 \\
8 & 24 \\
9 & 27 \\
10 & 30.1 \\
11 & 33.1 \\
12 & $\underline{36.1}$ \\
\hline
\end{tabular}

بـا توجـهـ بـه معلوم بودن همـهـ متغيرهـا بـهـ جز متغير حـداكثر برد اندازهزيرى، مىتوان رابطه 1 را به صورت رابطه (9) ساده كرد:

$20 \log R_{\max }+\alpha R_{\max }=y$

در نتيجه حداكثر برد اندازهگيرى در شرايط ايدهآل از رابطه ( •() قابل محاسبه است:

$R_{\text {max }}=e^{\left.\frac{\ln (10)\left(y-\frac{20 \text { Lambertw }\left(\frac{\alpha e^{y \ln (10) \ln (10)}}{20}\right)}{\ln (10)}\right)}{20}\right)}$

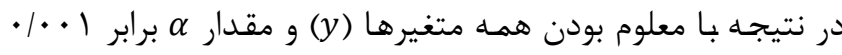

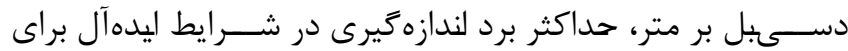

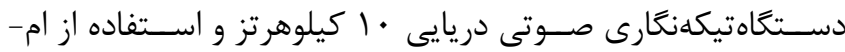

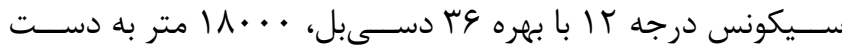
مى آيد. دستخاههاى تيكدنگارى صوتى دريايى قابليت اندازهخيرى سرعت جريان در هر •r ثانيه را دارند و براى افزايش دقت اندازهزيرى سرعت، تعداد • r داده را با ميانگين گيرى متحرك محاسبه مى كنند.

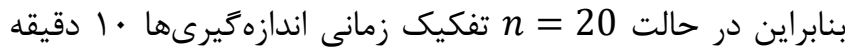
خواهد بود. با فرض ثابت بودن سرعت صوت برابر با • لها متر بر

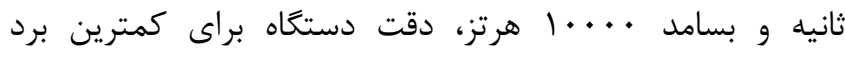

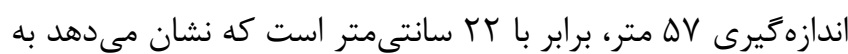
كارگيرى دستخاه تيكهنغارى صوتى دريايى • ا كيلوهرتز در فواصل كوتاه داراى دقت يايينى است. اين درحاليست كه با افزايش فاصله

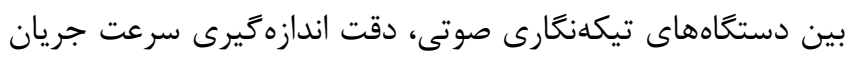




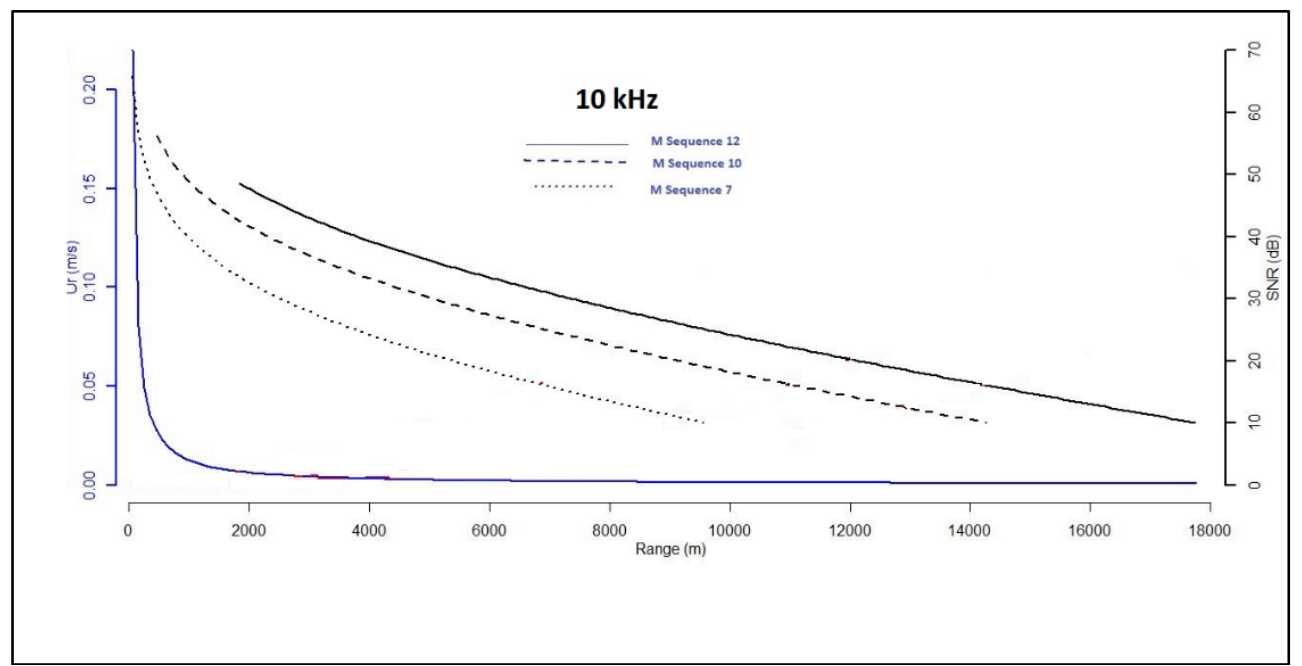

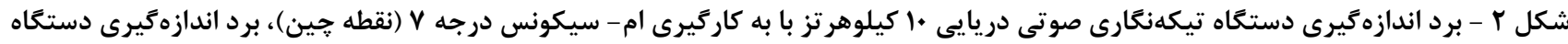

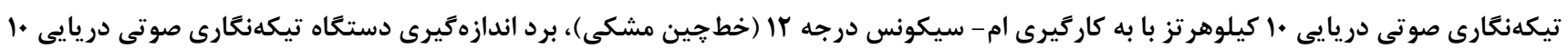

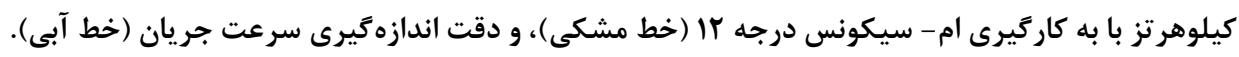

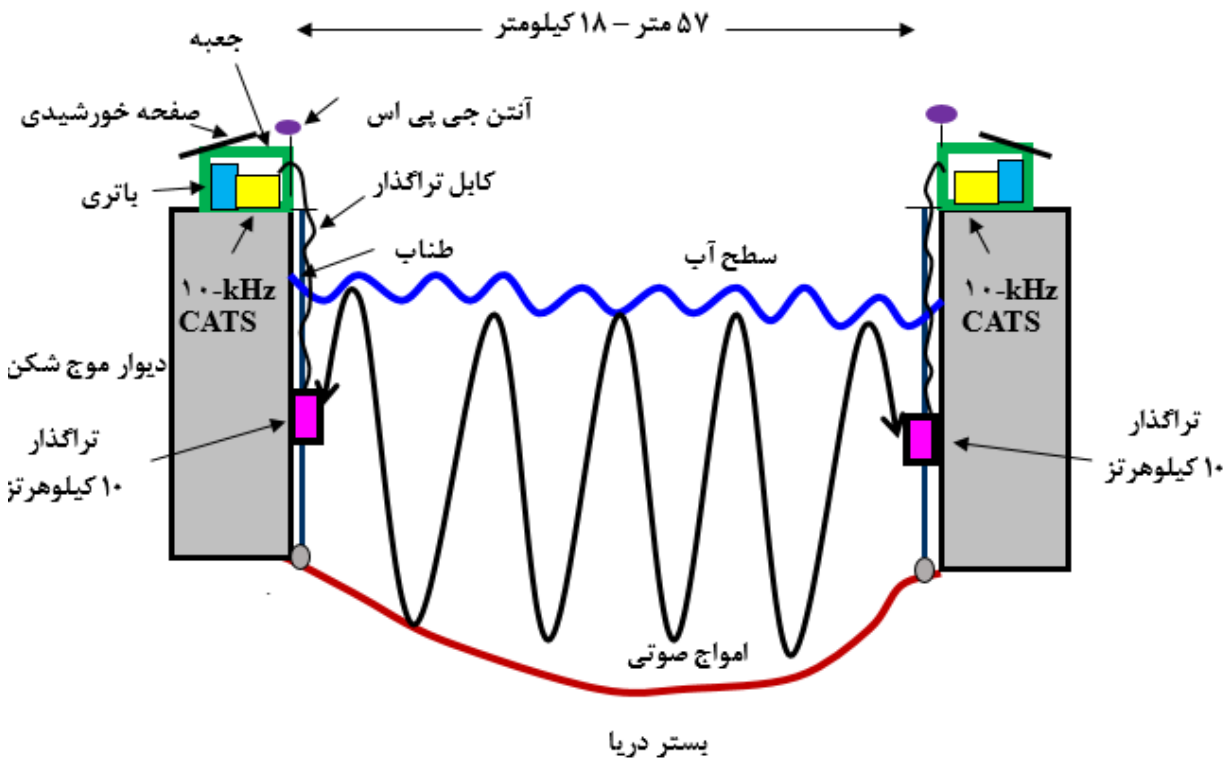

شكل r - مدل مفهومى بهكاركيرى دستخًاهتيكهنعارى صوتى دريايى •ا كيلوهر تز.

شايان ذكر است در طبيعت عوامل متعددى نظير وجود رسوبات معلق در آب، نوفههاى كشتى ها و آبزيان، طوفان و غيره سبب كاهش برد اندازهزيرى مىشود. از اين روى، بررسى مطالعات ييشين نشان

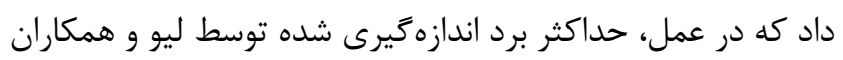

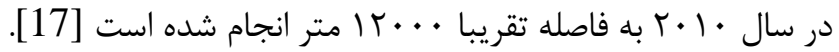
با توجه به هزينههاى بالاى اندازهيرىهاى ميدانى با ساير روشها

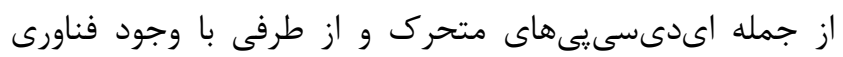
تيكدنكارى صوتى در ايران، بهكارگيرى اين روش بهمنظور رِايش جريانهاى دريايى در خليج فارس و درياى عمان توصيه مىشود. p

در اين مطالعه ارزيابى دستخاه تيكهنگارى صوتى دريايى . كيلوهرتز با رويكرد محاسبه حداقل و حداكثر برد اندازهيرى و و ارزيابى دقت اندازهيرى سرعت جريان آب در فواصل مختلف مورد

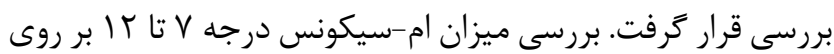
برد اندازهزيرى نشان داد كه فواصل QV متر تا هداكثر كمتر از

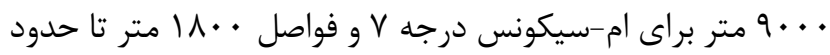

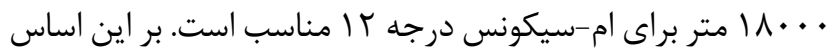
در شرايط ايدهآل با فرض عدم وجود ذرات معلق در آب، حداقل و

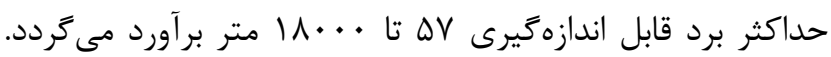


3- Bahreinimotlagh, M., Roozbahani, R., Eftekhari, M., Zareian, M. J. and Farokhnia, A., (2019), Evaluation of underwater acoustic propagation model (Ray theory) in a river using Fluvial Acoustic Tomography System, Journal of Acoustical Engineering Society of Iran, Vol. 6, p.29-38, [In Persian].

4- Baggeroer, A. and Munk, W., (1992), The Heard Island Feasibility Test, Physics Today, Vol. 45, p.22-30.

5- Taniguchi, N., Kaneko, A., Yuan, Y., Gohda, N., Chen, H., Liao, G., Yang, C., Minamidate, M., Adityawarman, Y., Zhu, X. and Lin, J., (2010), Long-term acoustic tomography measurement of ocean currents at the northern part of the Luzon Strait, Geophysical Research Letters, Vol. 37,.

6- Chen, M., Syamsudin, F., Kaneko, A., Gohda, N., Howe, B. M., Mutsuda, H., Dinan, A. H., Zheng, H., Huang, C.-F., Taniguchi, N., Zhu, X., Adityawarman, Y., Zhang, C. and Lin, J., (2018), Real-Time Offshore Coastal Acoustic Tomography Enabled With MirrorTranspond Functionality, IEEE Journal of Oceanic Engineering, p.1-11.

7- Syamsudin, F., Chen, M., Kaneko, A., Adityawarman, Y., Zheng, H., Mutsuda, H., Hanifa, A. D., Zhang, C., Auger, G., Wells, J. C. and Zhu, X., (2017), Profiling measurement of internal tides in Bali Strait by reciprocal sound transmission, Acoustical Science and Technology, Vol. 38, p.246-253.

8- Al Sawaf, M. B., Kawanisi, K., Kagami, J., Bahreinimotlagh, M. and Danial, M. M., (2017), Scaling characteristics of mountainous river flow fluctuations determined using a shallow-water acoustic tomography system, Physica A: Statistical Mechanics and its Applications, Vol. 484, p.11-20.

9- Bahreinimotlagh, M., Kawanisi, K., Danial, M. M., Al Sawaf, M. B. and Kagami, J., (2016), Application of shallow-water acoustic tomography to measure flow direction and river discharge, Flow Measurement and Instrumentation, Vol. 51, p.30-39.

10- Bahreinimotlagh, M., Kawanisi, K., Sawaf, M. ., Roozbahani, R., Eftekhari, M. and Kazemi Khoshuie, A., (2019), Continuous Streamflow Monitoring in Shared Watersheds Using Advanced Underwater Acoustic Tomography System: A Case Study on Zayanderud River. Environmental Monitoring Assessment.

11- Munk, W. and Wunsch, C., (1979), Ocean Acoustic Tomography: A Scheme for Large Scale Monitoring, Deep Sea Research Part A.

$$
\begin{aligned}
& \text { همجنين اين روش براى اندازهيرى جريان در رودخانهاى عريض } \\
& \text { جزر و مدى قابل استفاده است [24]. }
\end{aligned}
$$

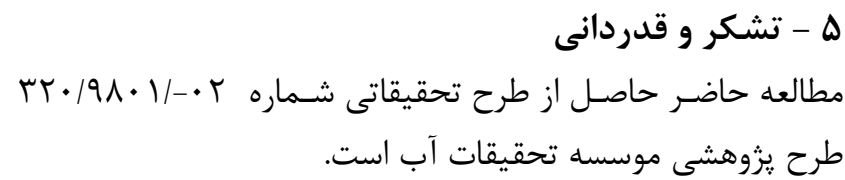

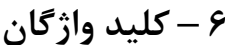

1- Acoustic Tomography Technique

2-ADCP (Acoustic Doppler Current Profiler)

3-AVM (Acoustic Velocity Meter)

4-Ocean Acoustic Tomography System

5-Coastal Acoustic Tomography Technique

6-Seto Inland Sea

7-Kanmon Strait

8-Data Assimilation

9-POM (Princeton Ocean Model)

10-Zhitouyang Bay

11-Inverse Problem

12-Coastal upwelling

13-Diurnal internal tides

14-Bachimen Harbor

15-Horizontal ADCP

16-M Sequence

17-Ambient noise

18-Digit

19-Q - value

20-Propagation Loss (PL)

21-Spreading Loss

22-Absorption Loss

23-Directivity

24-Reflection

25-Interference

26-RL (Receive Level)

27-Sound Level dB relative to $1 \mu \mathrm{Pa}$ at $1 \mathrm{~m}$.

28-Signal to Noise ratio

$$
\text { مراجع -V }
$$

1- Bahreinimotlagh, M., Roozbahani, R., Eftekhari, M., Heydari, A. K. and Abolhosseini, S., (2019), Investigation of Current Status in Haftbarm Lake Using Acoustic Tomography Technology, Journal of Water and Soil, Vol. 33, p.[In Persian].

2- Bahreinimotlagh, M., Roozbahani, R., Farokhnia, A., Soltaniasl, M. and Mohtasham, M., (2019), Technical Note: Acoustic Tomography Technology; a Useful Tool for Continuous Monitoring of Flow Velocity and Temperature, Iran-Water Resources Research, Vol. 14, p.271-275, [In Persian]. 
مسعود بحرينى مطلق و همكاران/ امكانسنجى بايش جريانهاى خليجفارس با استفاده از فناورى تيكهنغارى صوتى دريايى • ا كيلوهرتز

internal tides in Hiroshima Bay, Japan Chuanzheng, Journal of Geophysical Research: Oceans Research, p.1152-1172.

19- Huang, C.-F., Taniguchi, N., Chen, Y.-H. and Liu, J.-Y., (2016), Estimating temperature and current using a pair of transceivers in a harbor environment, Journal of Acoustical Society of America, Vol. 140, p.EL137EL142.

20- Bahreinimotlagh, M., Roozbahani, R., Eftekhari, M., Kardanmoghadam, H. and Kavousi, A., (2018), Design, Manufacture and the Evaluation of Fluvial Acoustic Tomography System (FATS), Journal of Acoustical Engineering Society of Iran, Vol. 6, p.1-11 [In Persian].

21- Bahreinimotlagh, M., Roozbahani, R., Eftekhari, M., Kardanmoghadam, H., Abbasi, M. and Mohtasham, K., (2019), Feasibility study of Fluvial Acoustic Tomography System for flood monitoring and determination of the measurement accuracy, minimum and maximum measurement ranges, Iranian Journal of Echo Hydrology, Vol. 6, p.585-592 [In Persian].

22- Urick, R. J., (1983). Principles of underwater sound, Peninsula Pub, New York.

23- Yamaguchi, K., Lin, J., Kaneko, A., Yayamoto, T., Gohda, N., Nguyen, H. Q. and Zheng, H., (2005), A continuous mapping of tidal current structures in the kanmon strait, Journal of Oceanography, Vol. 61, p.283-294.

24- Bahreinimotlagh, M., Roozbahani, R., Eftakhari, M., Kardan Moghaddam, H. and Hassanli, S. A., (2019), Continuous Monitoring of Tidal Bores Using Acoustic Tomography Technique, Journal of Oceanography, Vol. 9, p.57-64 [in Persian].
Oceanographic Research Papers, Vol. 26, p.123-161.

12- Howe, B. M., Worcester, P. F. and Spindel, R. C., (1987), Ocean acoustic tomography: Mesoscale velocity, Journal of Geophysical Research: Oceans, Vol. 92, p.3785-3805.

13- Yun Shen, Zhang, C., Huang, L., Zhang, C., $\mathrm{Wu}$, Y., Wu, G., Sheng, C., Guo, Y., Wang, Z., Liu, X. and Huang, H., Flow Velocity and Temperature Measuring in Large- Scale Wave-Current Flume by Coastal Acoustic Tomography, OCEANS 2018 MTS/IEEE, 2018, Charleston, SC, USA.

14- Zheng, H., Noriaki, G., NOGUCHI, H., Ito, T., Yamaoka, H., Tamura, T., Takasugi, Y. and Kaneko, A., (1997), Reciprocal Sound Transmission Experiment for Current Measurement in the Seto Inland Sea, Japan, Journal of Oceanography, Vol. 53, p.117-127.

15- Zheng, H., Yamaoka, H., Gohda, N., NOGUCHI, H. and Kaneko, A.; (1998), Design of the acoustic tomography system for velocity measurement with an application to the coastal sea, J. Acoust. Soc. Jpn. (E), Vol. 19, p.199-210.

16- Lin, J., Kaneko, A., Gohda, N. and Yamaguchi, K., (2005), Accurate imaging and prediction of Kanmon Strait tidal current structures by the coastal acoustic tomography data, Geophysical Research Letters, Vol. 32, p.1-4.

17- Liao, G., Wang, J., Xu, X., Yang, C., Wu, Q., Zhang, C. and Zhu, X., A Coastal Acoustic Tomography Inverse Method Based on Chebyshev Polynomials and Its Application in Zhoushan Field Experiment.

18- Zhang, C., Kaneko, A. ;, Xiao-Hua Zhu; and Gohda, N., (2015), Tomographicmapping of a coastal upwelling and the associated diurnal 